\title{
Understanding and Control of High Temperature Oxidation Flaws of Low-Density Poly(ethylene) with Raman Spectroscopy
}

\author{
Marie Veitmann, ${ }^{1,2,3}$ Richard Jumeau, ${ }^{1,2,3}$ Patrice Bourson, ${ }^{2,3}$ \\ Michel Ferriol, ${ }^{2,3}$ and François Lahure ${ }^{1}$ \\ ${ }^{1}$ Total Raffinage Chimie, Plateforme de Carling-Saint Avold, 57508 Saint-Avold, France \\ ${ }^{2}$ Laboratoire Matériaux Optiques Photonique et Systèmes (LMOPS), Université de Lorraine, 57070 Metz, France \\ ${ }^{3}$ Supélec, Laboratoire Matériaux Optiques Photonique et Systèmes (LMOPS), 57070 Metz, France \\ Correspondence should be addressed to Patrice Bourson; bourson@univ-metz.fr
}

Received 26 January 2014; Revised 17 March 2014; Accepted 3 May 2014; Published 5 June 2014

Academic Editor: Rolf W. Berg

Copyright (C) 2014 Marie Veitmann et al. This is an open access article distributed under the Creative Commons Attribution License, which permits unrestricted use, distribution, and reproduction in any medium, provided the original work is properly cited.

\begin{abstract}
Studies of high temperature oxidation of polyethylene are not much present in the literature though it can really be a problem especially in polymer production and processing. This study aims to detect oxidation flaws in polyethylene and to determine their impact on polymer structure and properties. Besides, we suggest a method via PLS-regression to determine the degree of flaws that can occur during polymer processing due to oxidation. Several kinds of oxidation flaws were reproduced in laboratory at $150^{\circ} \mathrm{C}$ in an oven operating in air and Raman spectroscopy analysis was performed on each sample. Using statistical tools as chemometrics on these spectra, we have built a Partial Least Square (PLS) model able to predict the oxidation degree of flaws. Interpretation of the model construction and further characterization tests show that oxidation can be followed with the evolution of the crystalline carbon group and of the created carbonyl functions. Finally we suggest possible mechanisms which can explain the high temperature oxidation process in LPDE, and we link them to the modification of the material properties.
\end{abstract}

\section{Introduction}

Low-density polyethylene (LDPE) is the most popular used thermoplastic polymer in the world, with a wide range of applications, particularly in flexible packaging. According to the Global Business Intelligence Research [1], the European LDPE demand in 2013 was close to 10 million Metric Tons. LDPE is usually produced by high-pressure radical polymerization.

In this process, two kinds of flaws can occur during polymerization and thus are problematic for LDPE manufactures. The so-called "molten flaws" consist of a mix of two kinds of polymers with different Melt Flow Index (MFI). This is not a real problem if they are present in small quantities because most of the time they will melt and disappear during polymer processing. On the contrary, the "unmolten flaws" such as cross-linking or oxidation affect the polymer structure. The presence of these flaws during production will lead to a real weakness for polymer processing. Ability to identify and quantify these specific flaws is thereby a major issue in polymer industry. By combining Raman spectroscopy and chemometrics, this study aims to suggest a method to determine the amount of flaws due to oxidation occurring during polymer processing.

On one hand, the rapidity and easiness of Raman spectroscopy make it the ideal candidate for industrial control. Moreover, Raman spectra offer a wide range of information on the analyzed polymer. Many studies report that use of Raman spectra of semicrystalline polyethylene was promising in elucidating structural and morphological state [2] such as crystallinity characterization [3] or density determination [4]. Use of such a technique allows thereby accessing different polymer properties useful to control production with only one experiment and without sample preparation.

On the other hand, the oxidative degradation of polyolefins can be followed by measurement of the level of carbonyl group absorption by infrared spectroscopy (FTIR) [5]. Similar tests were performed on samples oxidized at high 
temperature in order to recreate the manufacturing conditions.

In the same way as Skagerberg et al. who demonstrated how the Partial Least Square (PLS) method can be used to perform quality control and Statistical Process Control (SPC) from one section of a LDPE reactor [6], PLS-regression was used to create a model able to recognize and quantify oxidation of the samples.

Finally, thanks to the model construction and further characterization tests, the interpretation of Raman spectra led to the establishment of high temperature oxidation patterns.

\section{Experimental}

2.1. Materials. Viscous low-density polyethylene (LDPE) was investigated in this study. Two batches were prepared. The first was obtained by melting at $150^{\circ} \mathrm{C}$ in a drying oven 30 grams of LDPE pellets in an alimentary silicone cupcake mold and treated during 492 hours. The second set was composed of ten thin disks ( $700 \mu \mathrm{m}$ in thickness) obtained by pressing under 4 bars 1 gram of molten pellets at $150^{\circ} \mathrm{C}$.

All the disk samples were oxidized in air at $150^{\circ} \mathrm{C}$ during 1 to 591 hours and then cooled in a refrigerator set to $2^{\circ} \mathrm{C}$ during 2 minutes to obtain a homogeneous and fast cooling.

In order to observe the depth of oxidation in the "polymer cake," the samples were cut into slices of 2-centimeter width and polished with sandpaper of different sizes (150, 300, 600, and $1200 \mathrm{gr} / \mathrm{inch}^{2}$ ). The coloration depth was observed on "polymer cake" slice using an optical microscope with $\times 24$ magnification (Motic China Group Co., Ltd). Linear change of coloration with the depth is correlated with the penetration of oxidation. Polymer cakes are thus heterogeneous in depth.

\subsection{Methods}

2.2.1. Raman Spectroscopy. Raman spectra were recorded using a HORIBA JobinYvon LabRAM ARAMIS fitted with a $785 \mathrm{~nm}$ laser and a $250 \mu \mathrm{m}$ confocal pinhole. The instrument was calibrated using a silica sample prior to the experiments. All spectra on disks were obtained in the spectral range 800$3000 \mathrm{~cm}^{-1}$ with an integration time of 30 seconds and at least three repetitions per location. The laser was focused using a $\times 100$ objective and at least four spectra were performed on each disk.

Raman mapping on the "polymer cake" slice was performed in the spectral range $1363-1820 \mathrm{~cm}^{-1}$ with the same integration time but only two repetitions. The sample was positioned on a $X Y$ micrometer stage that could also be vertically moved in the $Z$ direction and the laser was focused with a $\times 10$ objective to collect spectra across the slice. The point-to-point distance between each spectral collection was about $50 \mu \mathrm{m}$. Data acquisition was performed using HORIBA LabSpec 5 software.

Chemometrics operations were carried out using OPUS version 7.0 software from Bruker Optic. Partial Least Square (PLS) regression was used for the whole study and a crossvalidation procedure was used. All spectra were pretreated thanks to a Standard Normalization Vector (SNV) treatment to correct effects of the multiplicative interferences of scattering. The treatment consisted in subtracting the mean spectrum and scaling with the spectrum standard deviation [7]. Processed spectra were fitted using Fityk 1.2 software and the different peaks were attributed to the corresponding organic groups [8].

2.2.2. Other Characterization Techniques. Infrared spectroscopy measurements were performed with a Nicolet Impact 410 FTIR device. Five Attenuated Total Reflectance (ATR) spectra were collected on each disk using 32 scans and a $4 \mathrm{~cm}^{-1}$ resolution.

WAXS measurements were carried out with a Bruker D8 Advance diffractometer. The diffraction pattern was integrated for $2 \theta$ between $5^{\circ}$ and $30^{\circ}$. The interval from $15^{\circ}$ to $28^{\circ}$ was used to calculate sample crystallinity. Both (110) and (200) reflections located, respectively, at $21.6^{\circ}$ and $24.0^{\circ}$ and the amorphous halo were fitted. The degree of crystallinity $X_{c}$ was calculated from the ratio of the area under the two crystalline peaks and of the sum of the area under the crystalline peaks and the amorphous halo (1) $[9,10]$ :

$$
X_{c}=\frac{\left(A_{(110)}+A_{(200)}\right)}{\left(A_{(110)}+A_{(200)}+A_{(\text {amorphous halo })}\right)} .
$$

All the obtained values were normalized with the value $X_{0}$ of the starting nonoxidized polymer to overcome the orientation effects due to the disk processing. Therefore, the relative crystallinity $X_{c} / X_{0}$ was used to follow the evolution of the crystallinity degree with thermal oxidation.

The crystallinity degree was confirmed by DSC measurements performed with a Netzsch DSC 200 F3 Maia instrument operating in nitrogen atmosphere. The annealing procedure consisted in two heating-cooling cycles from $20^{\circ} \mathrm{C}$ to $150^{\circ} \mathrm{C}$ with levels of 5 minutes at these temperatures to ensure that the samples were uniformly heated or cooled. The thermal curves were recorded at $10^{\circ} \mathrm{C} / \mathrm{min}$. Only the second heating run was used.

Tensile tests were performed through video traction [11] with a servohydraulic machine (MTS 810). The samples were cut from $70 \times 50 \mathrm{~mm}$ plates with a thickness of $3 \mathrm{~mm}$ and then oxidized in the same conditions as the disks. Tensile velocity was $5 \cdot 10^{-4} \mathrm{~s}^{-1}$ for each sample.

The density of samples was measured with a SARTORIUS LA2305 hydrostatic balance with ethanol as reference.

\section{Results and Discussion}

3.1. Oxidation Rate Prediction. The prediction of the oxidation time was based both on the measurement of Raman spectra on the surface of the disk samples oxidized during different times and on the realistic assumption that oxidation along the "polymer cake" thickness can be related to the oxidation time.

In this way, a first Raman investigation was performed on the surface of the ten disks to obtain a "time scale" with Raman spectra. At least four spectra per disk were recorded at different places on the surface. Figure 1(a) shows typical 


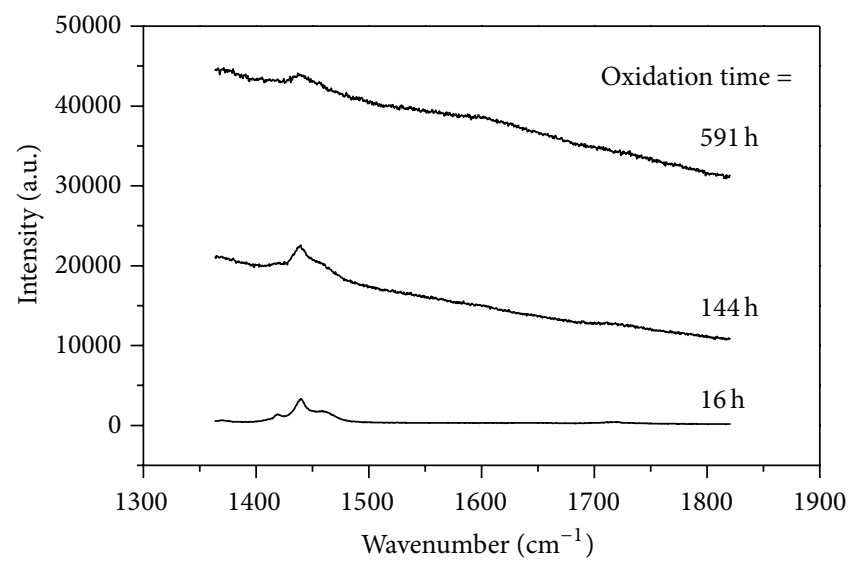

(a)

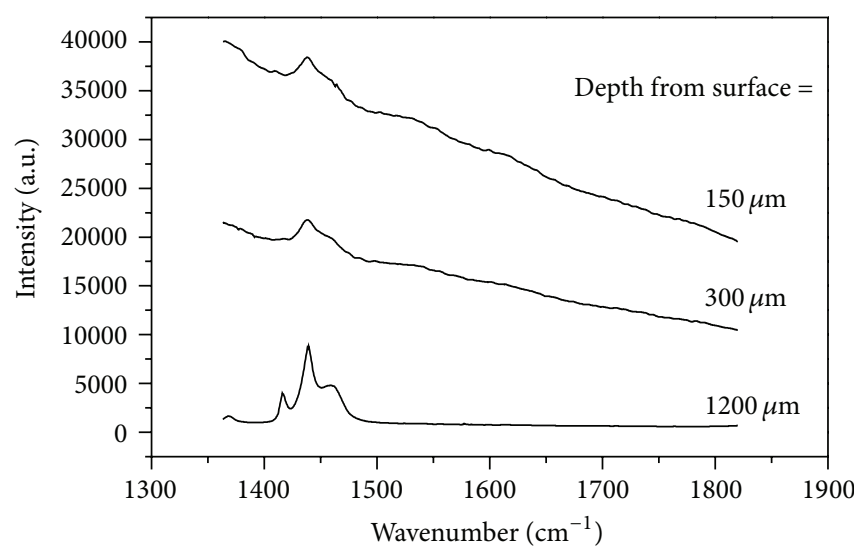

(b)

FIgURE 1: Raman spectra obtained on the disk's surface (a) and in the "polymer cake" depth (b).

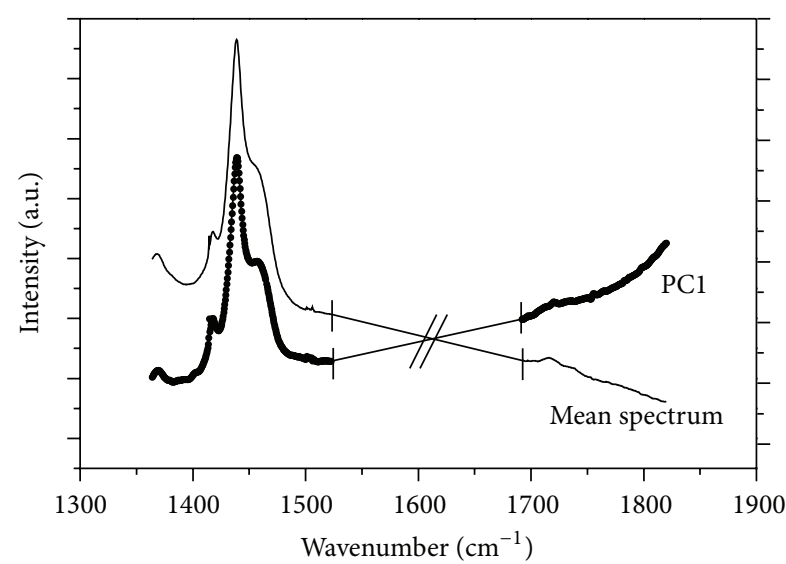

(a)

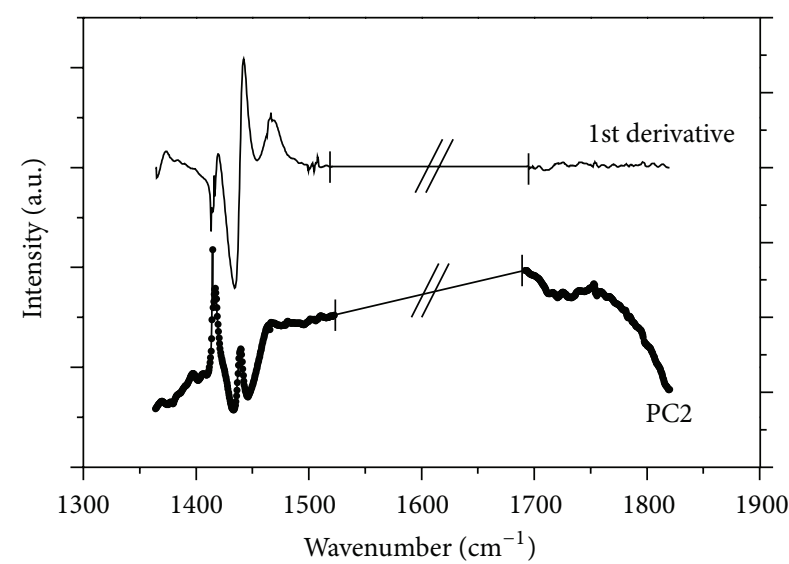

(b)

FIGURE 2: Factors used for PCA. First loading (a) is assimilated to the intensity of Raman spectra. The second loading (b) is correlated to the first derivative of the spectra.

spectra for different oxidation times. Moreover, a Raman mapping of the thickness of the "polymer cake" slice until a $2500 \mu \mathrm{m}$ depth was performed allowing associating each spectrum to an oxidation thickness. Figure 1(b) shows a series of three Raman spectra obtained for different depths. A luminescence phenomenon is observed because of the coloration due to oxidation which affects spectra processing. Chemometrics was used to overcome this phenomenon.

In a first step, we realized Principal Component Analysis (PCA) on the pretreated data collected from Raman mapping of the slices in order to explain the main variability of our Raman spectra. Results show differentiation of the spectra by two principal components presented in Figure 2. First loading is assimilated to the global intensity of the spectra, which translate an influence of oxidation degree on the spectral band intensity. Second loading is correlated to the first derivative of the spectra and therefore indicates shift of the peak. This shift translates the state of constraint to the oxidized materials.
In a second time, we choose this loading in Partial Least Square (PLS) regression to build a model able to predict the oxidation depth. Two other loadings were selected to obtain the best prediction. Moreover, only spectra for a depth between 0 and $1200 \mu \mathrm{m}$ were used because spectra beyond this value did not show any other evolution of the polymer. This limit was confirmed by the end of coloration as observed by optical microscopy (Figure 3 ). The model was developed by a 1-fold cross-validation using both spectral ranges $1690-1820 \mathrm{~cm}^{-1}$ and $1360-1520 \mathrm{~cm}^{-1}$. The resulting root mean standard error of cross-validation (RMSECV) was $27.4 \mu \mathrm{m}$ with the use of four factors. The predicted oxidation thickness by the PLS model as a function of real thickness is shown in Figure 4 (model 1). A similar result was obtained with two other "polymer cakes" made from the same LDPE pellets.

Third step was to predict a theoretical depth collected from the data of the surface of disk samples, oxidized during different durations, thanks to the PLS model. Each depth 


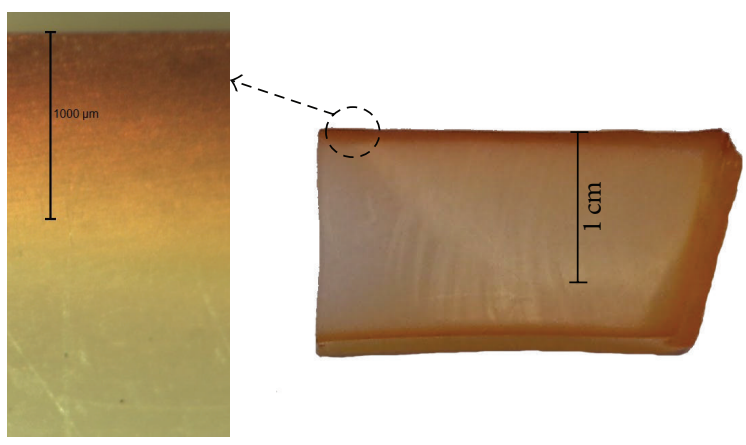

FIGURE 3: Micrographs of the polymer cake slice.

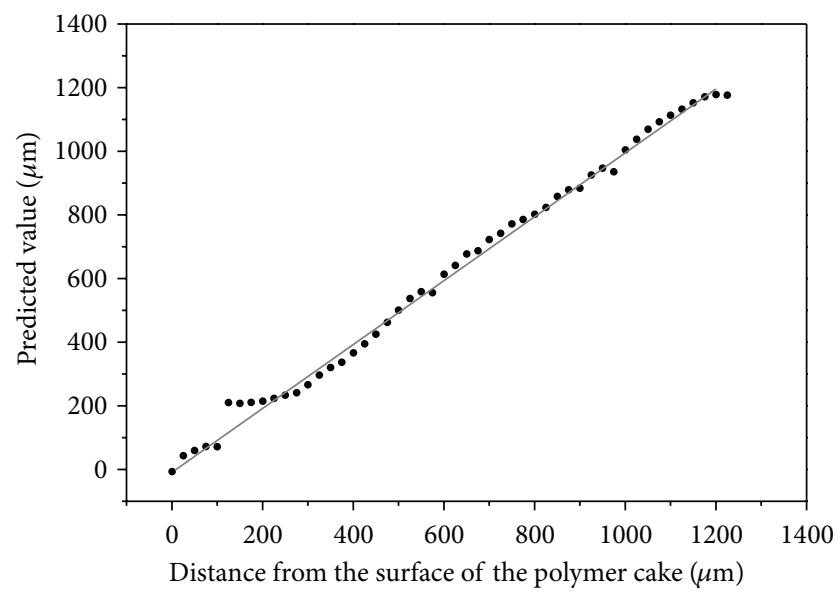

FIGURE 4: Validation of the PLS-model 1: Predicted oxidation thickness as a function of real depth.

value was then related to the oxidation time. The evolution of depth (distance from the surface of the polymer cake to the oxidation limit inside the sample) and oxidation time of the disks was fitted with an exponential regression as shown in Figure 5.

Finally, a second PLS-model (model 2) able to directly predict the oxidation time was established by translating depth values obtained on the Raman mapping spectra into time values using the previous exponential relation. An exponential format was used to build this new model. The same parameters as for the previous depth prediction model were used, 1-fold cross- validation using the same spectral ranges was done and the resulting RMSECV was 0.0329 with the use of three factors.

Model 2 was tested with all the disks spectra and the prediction was compared to the actual oxidation time (Figure 6). Due to the oxidation depth limit observed, predictions above this value were unable to be translated into an oxidation time because of the exponential relationship. However, it allows considering that the predicted oxidation degree is higher than the maximal oxidation degree used to build the model. We shall describe this result as a strong oxidation.

Nevertheless, although the predicted time does not exactly reflect the true oxidation time, it appears that one can recognize the oxidation degree of an analyzed sample. Thus,

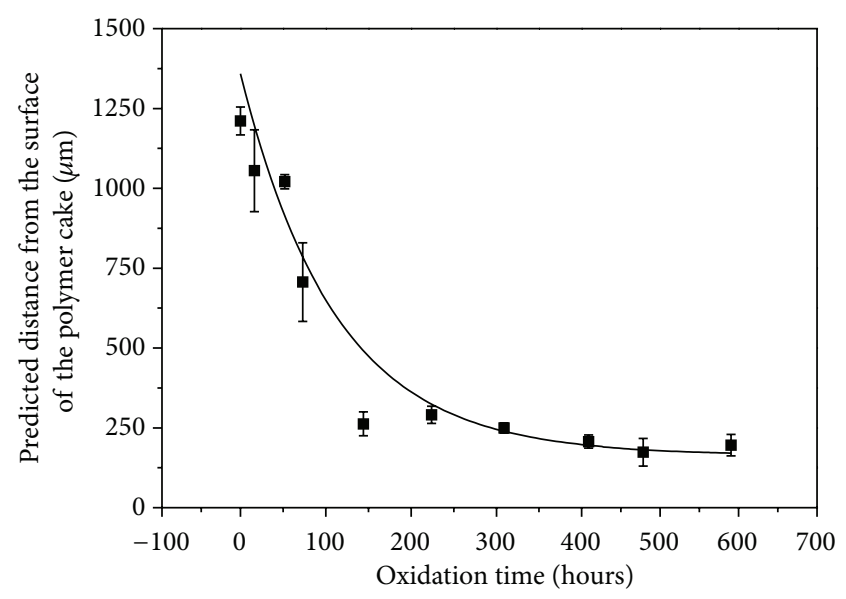

FIGURE 5: Relation between the distance from the surface to the oxidation limit of the polymer cake and oxidation time.

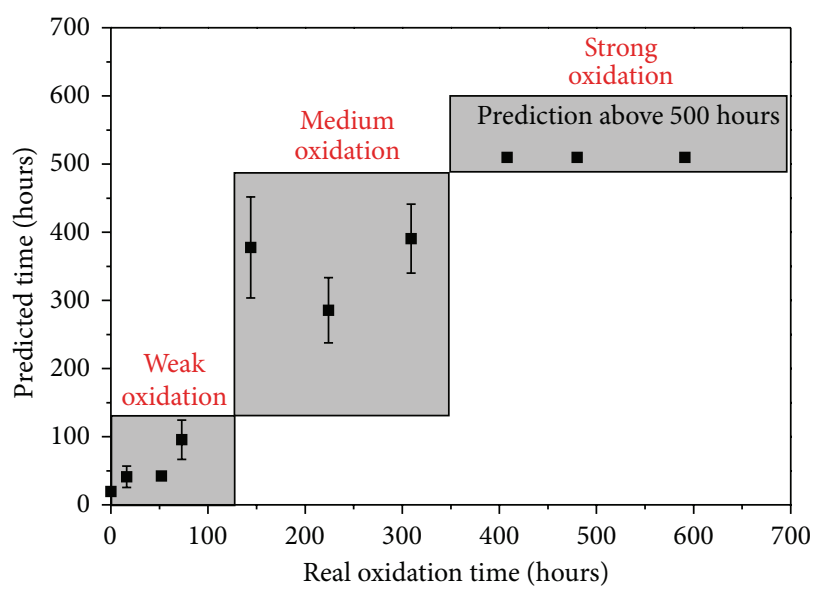

Figure 6: Validation of the PLS-model 2. All the disks spectra were tested with the model and the predicted oxidation time was compared with the real value allowing depicting three oxidation degrees: weak, medium, and strong.

on one hand, three degrees are perceivable: weak, medium, and strong oxidation depending on the predicted time. On the other hand, the real time oxidation was attributed to each oxidation degree by comparing the prediction of the disk spectra and their oxidation time. Thereby, we build a PLS-model 2, which is able to recognize oxidation on the analyzed sample and quantify it by oxidation degree, and also evaluate the time during which the polymer was in contact with oxygen for a given temperature.

3.2. Model Interpretation. Polymer structure modifications induced by oxidation are studied by the understanding of the loading used in the model. In order to perform this analysis, the two first loading vectors previously described in Figure 2 were studied.

It appears that to build the model, two spectral ranges are necessary and sufficient to have the more efficient prediction, 


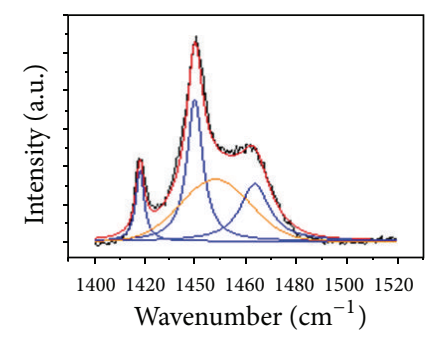

(a)

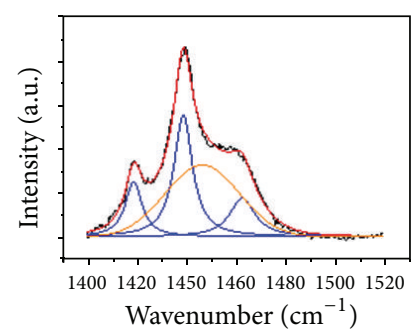

(b)

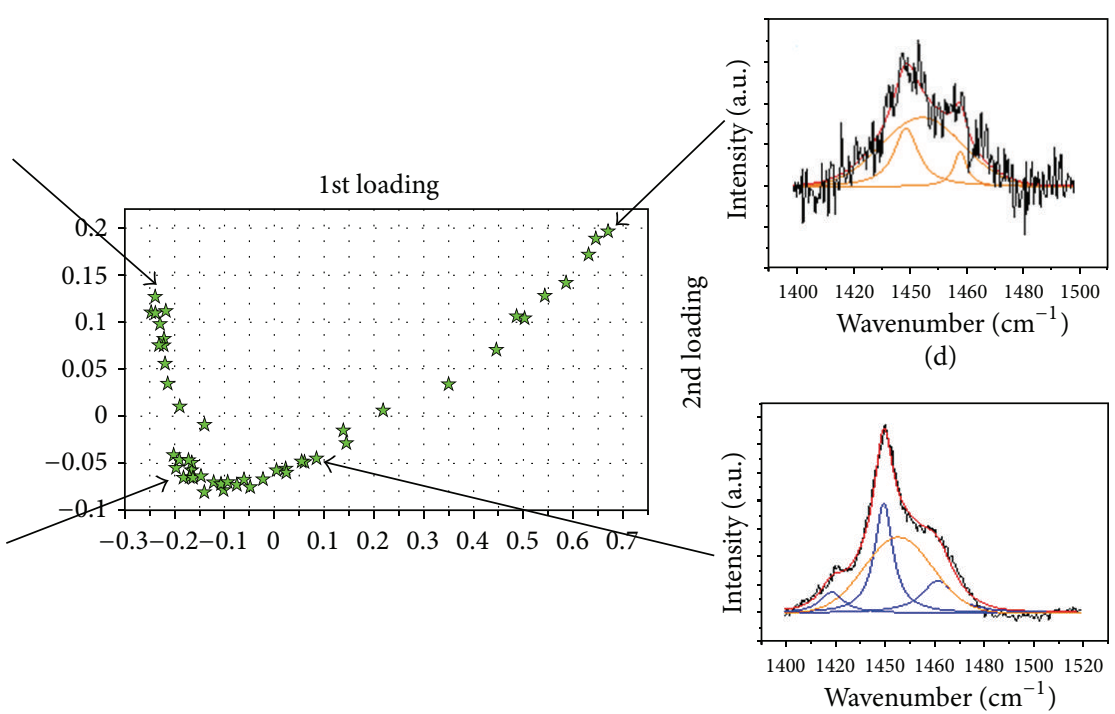

(c)

FIGURE 7: Evolution of the scores in function of the first and the second loading. Disk spectra corresponding to four scores are represented as a function of the oxidation degree: (a) nonoxidized, (b) weak, (c) medium, and (d) strong oxidation. The diminution in intensity of the Lorentzian peaks and the increase of the amorphous peaks show the impact of oxidation on the polymer crystallinity.

which means that species present in these spectral ranges are affected by the oxygen attack.

The first range from $1360 \mathrm{~cm}^{-1}$ to $1520 \mathrm{~cm}^{-1}$ can be attributed to $-\mathrm{CH}_{2}$ Raman vibration bands. In order to understand the modification of these species resulting from oxidation, a model was created using the same loading than the real model but only in the spectral range $1360-1520 \mathrm{~cm}^{-1}$. The evolution of the scores as a function of the two first loadings is shown in Figure 7. Raman spectra from one disk sample of each oxidation degree are associated with its corresponding score. Firstly, the model seems to differentiate oxidation depending on the second loading which, as previously seen, can be attributed to the peaks position. Weak oxidation is therefore characterized by the state of strain of the molecular chains. In a second time, scores are mostly classified thanks to the first loading corresponding to the intensity of the spectra. Medium oxidation is thus mainly identified by the concentration of each kind of bond. Finally, recognition of strong oxidation needs the combination of both loadings.

Raman spectra resulting from four of the scores are also represented in order to highlight the described spectral modifications. Spectra were fitted according to Jumeau et al. [12]: three Lorentzian peaks at 1420,1440 , and $1462 \mathrm{~cm}^{-1}$ corresponding to $\mathrm{CH}_{2}$ stretching of the crystalline phase and a Gaussian peak at $1440 \mathrm{~cm}^{-1}$ resulting from the amorphous region. Peaks position of weak and medium oxidation spectra was compared in Table 1 to the ones from the first score, which we considered as the reference in terms of peak intensity and position. Shift of the Gaussian peak is tallying with the previous observation and suppose that the amorphous phase is subject to elongation after the solidification following a high temperature oxidation. The intensity reduction of the two
TABLE 1: Vibrational bands frequencies in the spectral range 1400$1500 \mathrm{~cm}^{-1}$.

\begin{tabular}{llcl}
\hline & Reference & $\begin{array}{c}\text { Weak } \\
\text { oxidation }\end{array}$ & $\begin{array}{c}\text { Medium } \\
\text { oxidation }\end{array}$ \\
\hline $\begin{array}{l}\text { Lorentzian fitted } \\
\text { bands }\end{array}$ & $1418 \mathrm{~cm}^{-1}$ & $1419 \mathrm{~cm}^{-1}$ & $1419 \mathrm{~cm}^{-1}$ \\
& $1463 \mathrm{~cm}^{-1}$ & $1463 \mathrm{~cm}^{-1}$ & $1463 \mathrm{~cm}^{-1}$ \\
\hline $\begin{array}{l}\text { Gaussian fitted } \\
\text { bands }\end{array}$ & $1448 \mathrm{~cm}^{-1}$ & $1439 \mathrm{~cm}^{-1}$ & $1439 \mathrm{~cm}^{-1}$ \\
\hline
\end{tabular}

1420 and $1462 \mathrm{~cm}^{-1}$ peaks can be observed on all spectra and is the result of the decrease of the amount of crystalline phase. This result was confirmed by XRD and DSC experiments performed on the disks as shown in Figure 8.

Diminution and shift to lower temperature of the "endothermic" peaks initially present at $117^{\circ} \mathrm{C}$ demonstrate that crystal size reduces progressively with oxidation which leads to the diminution of the crystalline fraction. Thus, the PLS-regression is based, among others, on the crystalline changes to characterize oxidation.

The second spectral range used to build the PLS-model from $1637 \mathrm{~cm}^{-1}$ to $1820 \mathrm{~cm}^{-1}$ corresponds to the vibrational bands created with high temperature oxidation. Two areas seem to be impacted by oxidation, one from the spectral range $1640-1680 \mathrm{~cm}^{-1}$ which can be attributed to the carbon double bond vibrational band, and the spectral range from 1700 to $1750 \mathrm{~cm}^{-1}$ which is the result of carbonyl functions formation. Fitting by hand was hardly possible on the Raman spectra due to the luminescence phenomenon. FTIR measurements were performed on the disks to follow the evolution of these functions. Figure 9 presents some Infrared 


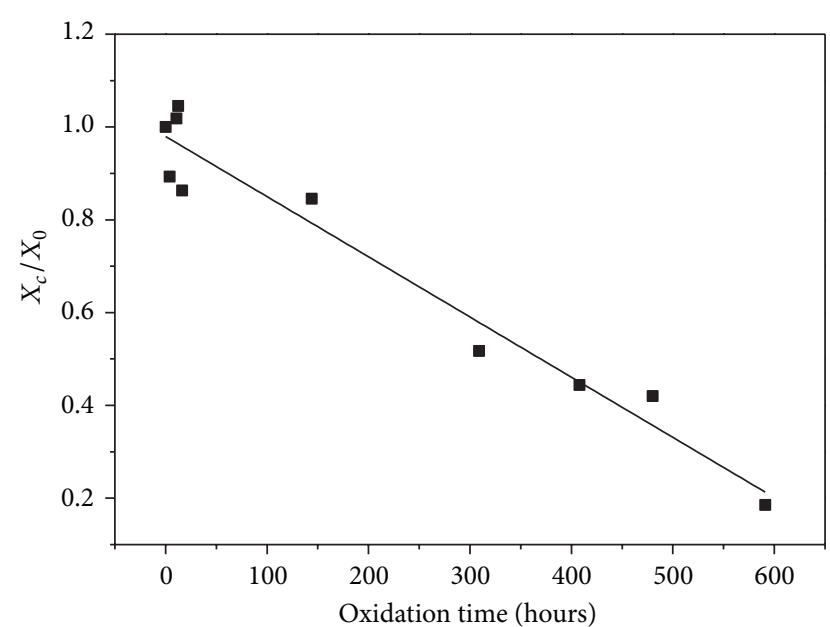

FIGURE 8: Diminution of crystallinity as a function of the oxidation time, measured by XRD experiments.

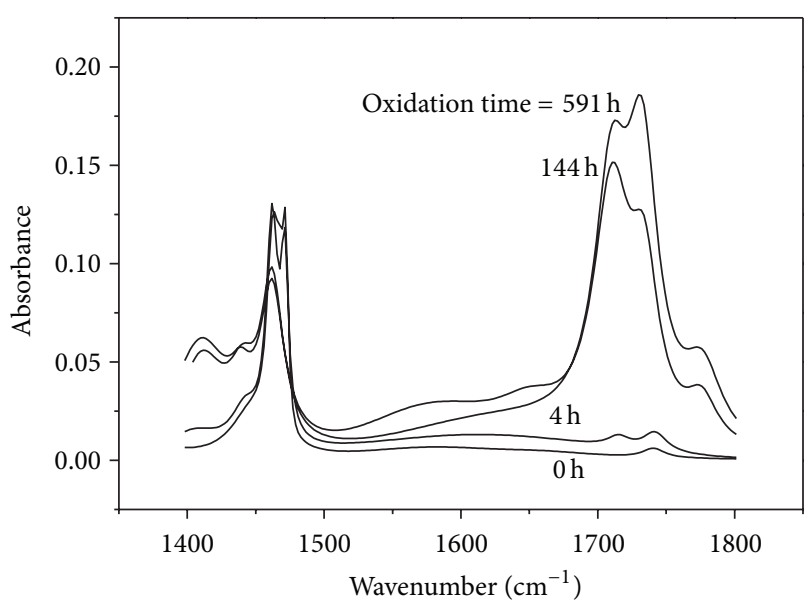

FIGURE 9: Fitted FT-IR spectra. Four oxidation times are drawn: a strong oxidation $(591 \mathrm{~h})$, a medium oxidation $(144 \mathrm{~h})$, a weak oxidation $(4 \mathrm{~h})$, and the reference.

spectra. Three carbonyl vibration bands appear with oxidation at $1714 \mathrm{~cm}^{-1}, 1733 \mathrm{~cm}^{-1}$, and $1775 \mathrm{~cm}^{-1}$, respectively, corresponding to ketone and aldehyde, ester, and aromatic ketone or other complicated species as keto-ester or oxalate [8]. These functions are also present on Raman spectra in the spectral range $1700-1750 \mathrm{~cm}^{-1}$ but with a very weak intensity. However, loadings presented in Figure 2 take into account this spectral signature. Thus, PLS-regression is also based on the recognition of these new functions created with high oxidation to identify oxidation flaws.

3.3. High Temperature Oxidation Pattern. Observations made during the model interpretation suggest by different ways that viscous polyethylene oxidation (high temperature oxidation) bring into play mechanisms different from solid polyethylene ageing. They can be explained by the different molecular configurations between the solid and the viscous polyethylene chains before oxygen attack. Indeed, no more

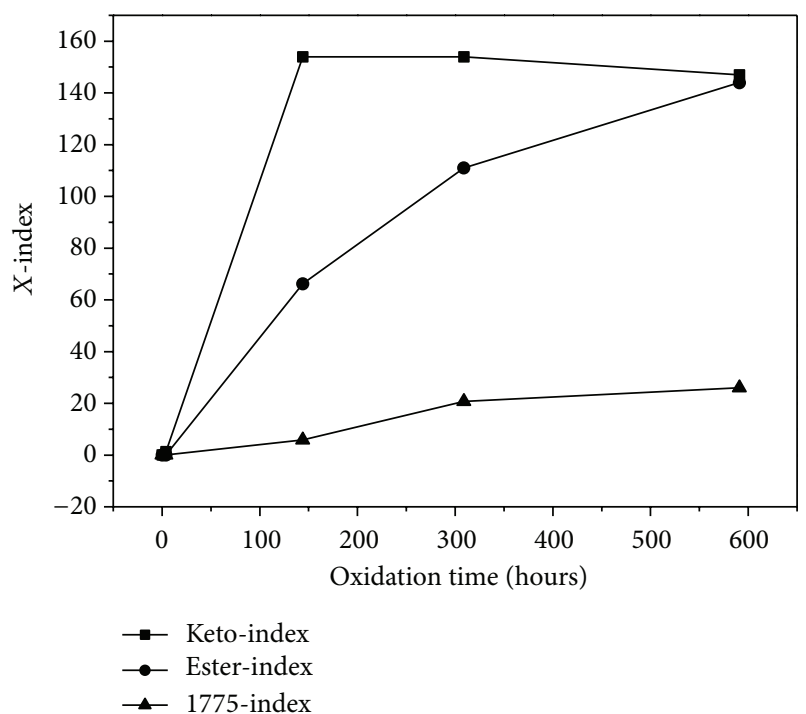

FIGURE 10: Development of oxidized species during oxidation. Each index was defined as the IR absorbance of the species normalized with the absorbance of the $-\mathrm{CH}_{2}$ group.

crystallized chain is present above the fusion temperature; every polymer subunits are oriented randomly with a statistical distribution of shape for all the chains. Thus, the whole molecule can be attacked by oxygen unlikely to solidified polyethylene where oxygen cannot reach the crystallized chains. This fact can explain the diminution of the crystalline phase during high temperature oxidation whereas crystallinity increases during a solid state oxidation. Moreover, the thermal agitation undergoes crosslinking of polymer chains [13]. This phenomenon will take an important part in oxidation reaction because it leads to the formation of carbon-carbon single bonds, which have a lower bonding energy than initially present carbon-hydrogen bonds. Thus, both the already present in branching base tertiary carbon bonds and the new bonds are the weakest of the molecule and will be preferential sites of oxygen attack.

Following the created oxidized species is possible by vibrational spectroscopy and gives good information about the different mechanisms which occur during viscous state oxidation. The evolution of the different oxidized species as a function of the oxidation time is plotted in Figure 10. In a same way as the team of Albertsson [5], keto-index, esterindex, and 1775-index were evaluated from the relative areas of each vibrational band to that of the methylene band at $1472 \mathrm{~cm}^{-1}$. First, keto-index sharply increases whereas 1775 index evolves in a nonmeaningful way. In a second time, the increase of the keto-index is stopped and the 1775index increases. Finally keto-index tends to decrease whereas the 1775-index no longer changes. Ester-index is constantly growing.

These observations lead to think that ketone and aldehyde are created at high concentration levels at the beginning and then once a certain concentration is reached, these oxidized are again attacked by oxygen to form a two-oxygen 


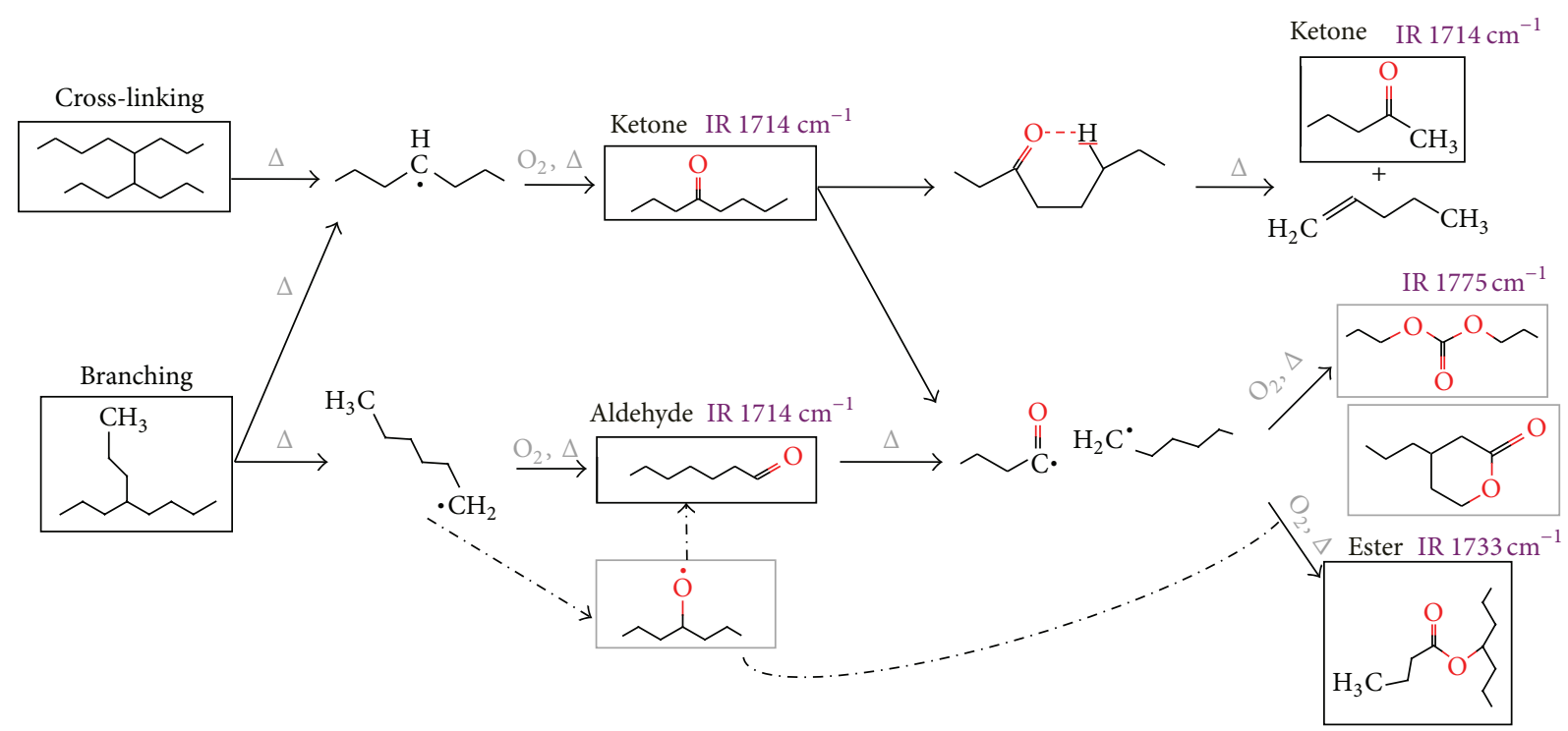

FIGURE 11: Suggested mechanisms of formation of the different observed patterns.

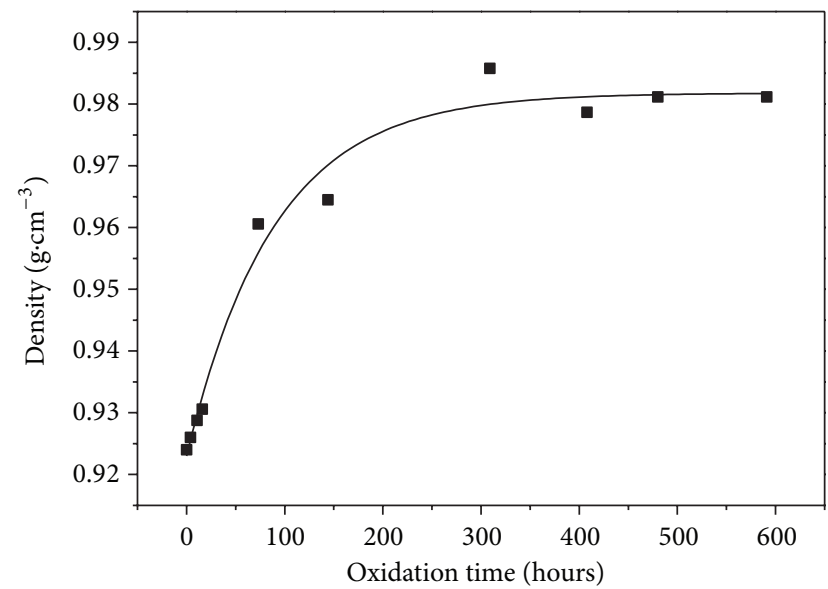

FIGURE 12: Evolution of density with oxidation.

function, as ester or keto-ester. Several recent works [5, 1416] especially about polyethylene degradation and photooxidation report some mechanisms likely to happen in high temperature oxidation. These mechanisms were adapted to our study with the help of our different observations and assumptions in order to establish the patterns of viscous state oxidation of polyethylene, presented in Figure 11. As assumed previously, oxidation will uppermost target branching and cross-linking. Thermal energy supplied during high temperature oxidation allows the creation of free radicals at the levels of the branching and the cross-linking which are the weakest bonds. Oxygen present in the air can then be absorbed by the macroradicals and form peroxides which undergo the formation of aldehydes and esters. Prolonged exposure may lead to the creation of new carbon and carboxyl radicals. Finally, rearrangement between all present radicals can lead to the formation of all different species observed

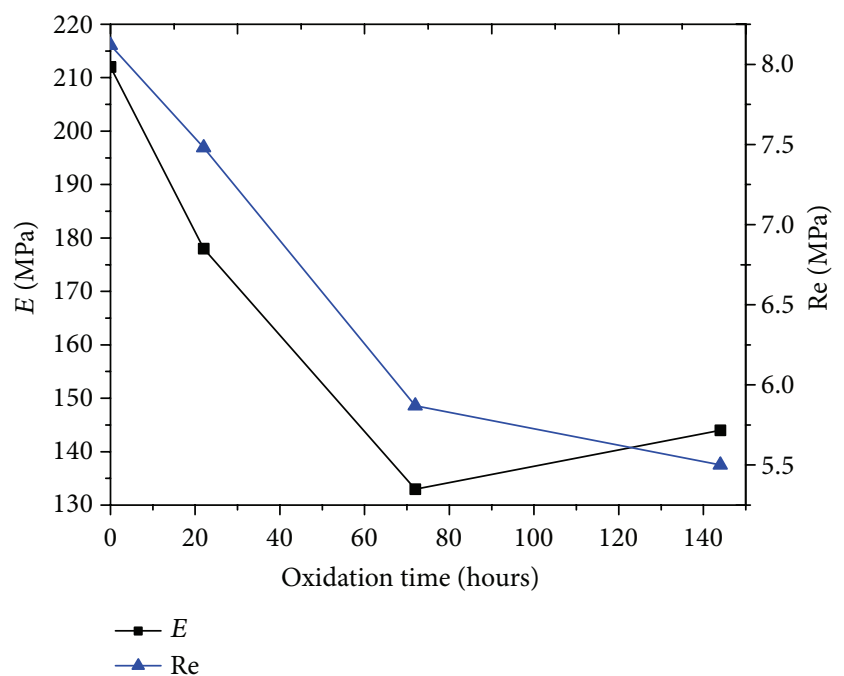

FIGURE 13: Evolution of the Young Modulus and yield strength of three oxidized samples.

by spectroscopy, as ester, $\beta$-keto-ester, $\alpha, \beta$-unsaturated fivemembered ring acid anhydride.

Other characterization tests were done in order to associate these structural changes and the polymer properties. Density calculation on the different disks, presented in Figure 12, shows an important increase of density until a few hundreds of hours, from which it remains constant. This time corresponds well to the asymptote observed in the relation between depth and oxidation time (Figure 4). This density increase could be explained by both crosslinking attack and the integration on the molecule of oxide. Stopped of this increase can be thereby translated by a stopped of the oxygen integration of the molecule, which means that the steady state is reached after few hundreds of hours for a $150^{\circ} \mathrm{C}$ 


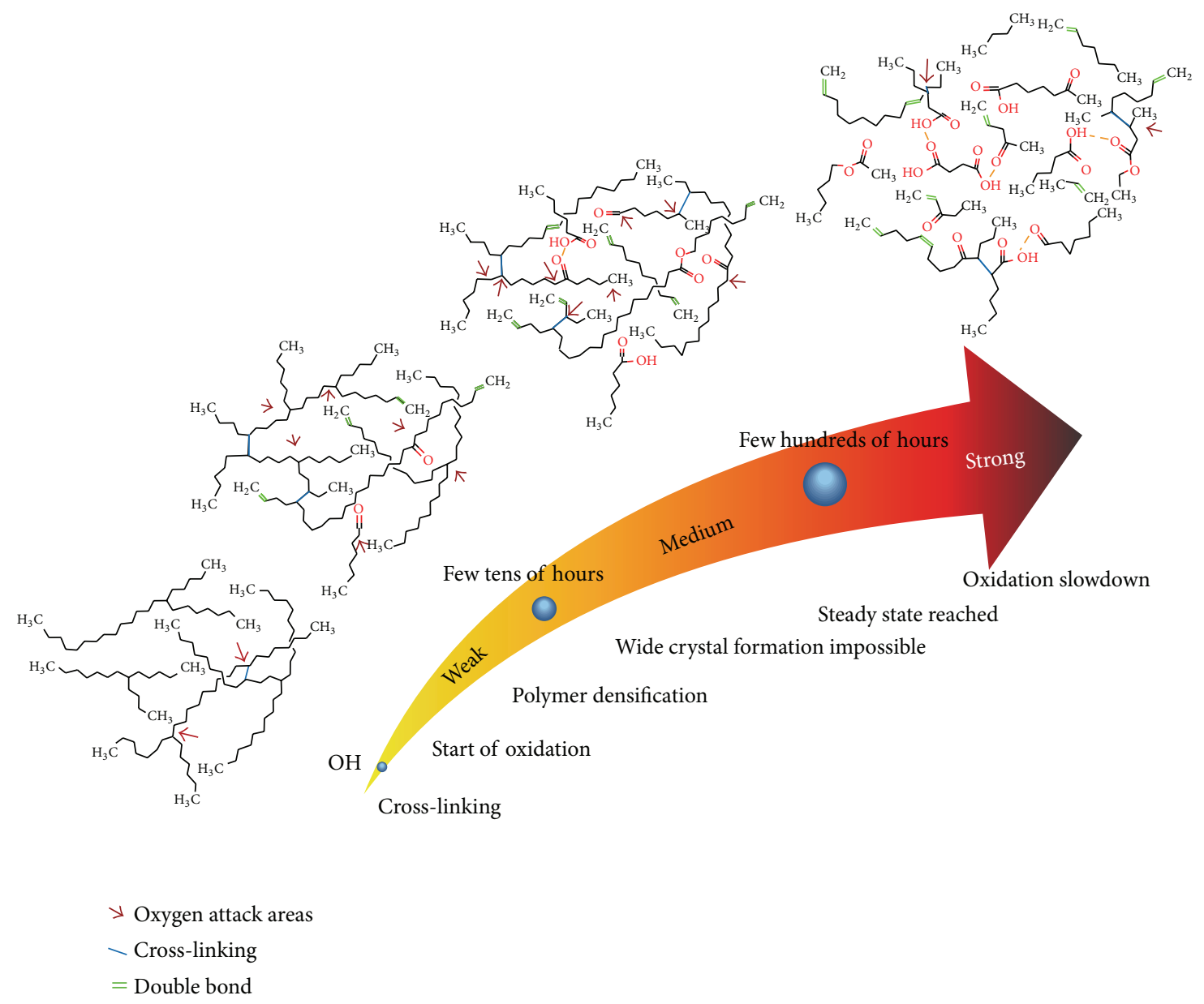

FIGURE 14: Impact of high temperature oxidation on LDPE chains.

oxidation. On the other hand, decrease of the crosslinking number also implies diminution of density. Both assumptions are supported by other observations, as the following of the ester-index or model construction.

On the other hand, tensile tests were made in order to follow oxidation impact on the polymer chains (Figure 13). The decrease of the Young modulus and the yield strength from 216 to $141 \mathrm{MPa}$ and 8 to $5,5 \mathrm{MPa}$ after 6 hours of oxidation, respectively, are an indicator of the chains cleavage. Impact of high temperature oxidation on LDPE chains is drawn in Figure 14. Representations of the different oxidation states are represented as a function of the impact on the polymer properties and can be condensed in four parts: initial stage, weak oxidation, medium oxidation, and strong oxidation.

\section{Conclusion}

This study develops a useful tool for controlling industrial production of polymers, that is to say, a method able to detect and classify oxidation flaws of low-density polyethylene with Raman spectroscopy. The model was built thanks to PLSregression with laboratory oxidized samples and successfully tested with real production flaws. Analysis of the regression construction shows that oxidation is detected with the structural change and the oxidized species formation observable on Raman spectra. Other characterization methods such as Infra-Red spectroscopy, DSC, XRD, or tensile tests have confirmed the structural change easily detected with Raman spectroscopy and allow knowing the structural state of the polymer as a function of the model prediction. Oxidation prediction tests made on the laboratory reproduced flaws highlight three oxidation degrees confirmed with the other characterization methods. Each degree can be distinguished from the other through both its oxidized species composition and its structural state.

Finally, interpretation to every characterization tests allowed proposing a possible mechanism of oxidation, which leads us to understand the impact of high oxidation on the polymer structure.

All these observations were made with $150^{\circ} \mathrm{C}$ oxidized samples, but other tests realized at $194^{\circ} \mathrm{C}$ suggest that this study can be adapted to other temperature.

\section{Conflict of Interests}

The authors declare that there is no conflict of interests regarding the publication of this paper. 


\section{Acknowledgments}

The authors would like to thank Total Raffinage Chimie for supporting this research project. The generous help of Professor Dahoun and Professor Royaud from the Institut Jean Lamour, France, is also gratefully acknowledged for their interest in this work and many useful discussions.

\section{References}

[1] PlasticsEurope, "Plastics: the facts 2013," 2013, http://www. plasticseurope.org.

[2] M. Glotin and L. Mandelkern, "A Raman spectroscopic study of the morphological structure of the polyethylenes," Colloid \& Polymer Science, vol. 260, no. 2, pp. 182-192, 1982.

[3] W. Lin, M. Cossar, V. Dang, and J. Teh, "The application of Raman spectroscopy to three-phase characterization of polyethylene crystallinity," Polymer Testing, vol. 26, no. 6, pp. 814-821, 2007.

[4] J. Kim, Y. Kim, and H. Chung, "Direct on-line Raman measurement of flying solid samples: determination of polyethylene pellet density," Talanta, vol. 83, no. 3, pp. 879-884, 2011.

[5] A.-C. Albertsson, S. O. Andersson, and S. Karlsson, "The mechanism of biodegradation of polyethylene," Polymer Degradation and Stability, vol. 18, no. 1, pp. 73-87, 1987.

[6] B. Skagerberg, J. F. MacGregor, and C. Kiparissides, "Multivariate data analysis applied to low-density polyethylene reactors," Chemometrics and Intelligent Laboratory Systems, vol. 14, no. 13, pp. 341-356, 1992.

[7] S. Roussel, Ondalys Formation, 2013.

[8] D. Lin-Vien, N. Colthup, W. Fateley, and J. Grasseli, The Handbook of Infrared and Raman Characteristic Frequencies of Organic Molecules, Academic Press, New York, NY, USA, 1991.

[9] S. Martín, J. F. Vega, M. T. Expósito, A. Flores, and J. MartínezSalazar, "A three-phase microstructural model to explain the mechanical relaxations of branched polyethylene: a DSC, WAXD and DMTA combined study," Colloid and Polymer Science, vol. 289, no. 3, pp. 257-268, 2011.

[10] R. A. Pereira, E. B. Mano, M. L. Dias, and E. B. Acordi, "Comparative study on the lamellar crystal structure of high and low density polyethylenes," Polymer Bulletin, vol. 38, no. 6, pp. 707714, 1997.

[11] C. G'Sell, J. M. Hiver, A. Dahoun, and A. Souahi, "Videocontrolled tensile testing of polymers and metals beyond the necking point," Journal of Materials Science, vol. 27, no. 18, pp. 5031-5039, 1992.

[12] R. Jumeau, P. Bourson, M. Ferriol, F. Lahure, M. Ponçot, and A. Dahoun, "Identification of LDPE grades focusing on specific $\mathrm{CH}_{2}$ Raman vibration modes," International Journal of Spectroscopy, vol. 2013, Article ID 720598, 6 pages, 2013.

[13] S. F. Chabira, M. Sebaa, and C. G'sell, "Influence of climatic ageing on the mechanical properties and the microstructure of low-density polyethylene films," Journal of Applied Polymer Science, vol. 110, no. 4, pp. 2516-2524, 2008.

[14] S. F. Chabira, M. Sebaa, and C. G'Sell, "Oxidation and crosslinking processes during thermal aging of low-density polyethylene films," Journal of Applied Polymer Science, vol. 124, no. 6, pp. 5200-5208, 2012.
[15] R. Maria, K. Rode, R. Brüll et al., "Monitoring the influence of different weathering conditions on polyethylene pipes by IRmicroscopy," Polymer Degradation and Stability, vol. 96, no. 10, pp. 1901-1910, 2011.

[16] M. Sudhakar, A. Trishul, M. Doble et al., "Biofouling and biodegradation of polyolefins in ocean waters," Polymer Degradation and Stability, vol. 92, no. 9, pp. 1743-1752, 2007. 

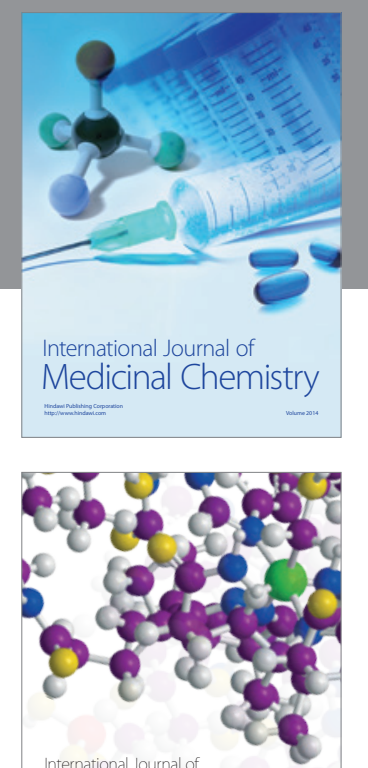

\section{Carbohydrate} Chemistry

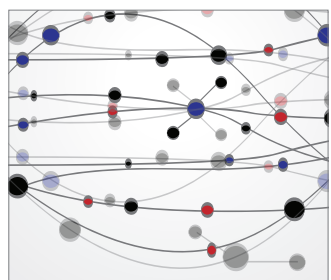

The Scientific World Journal
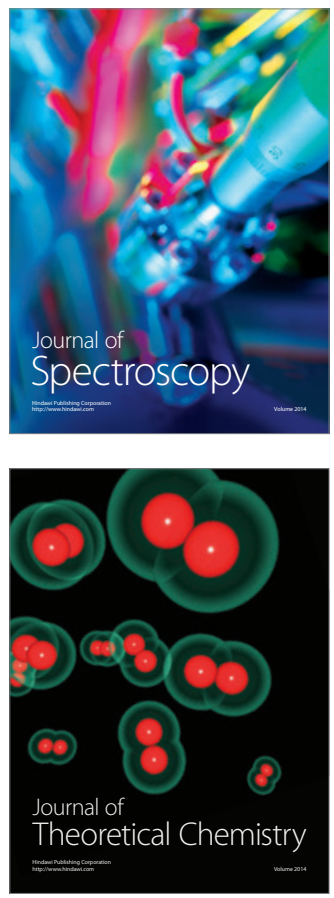
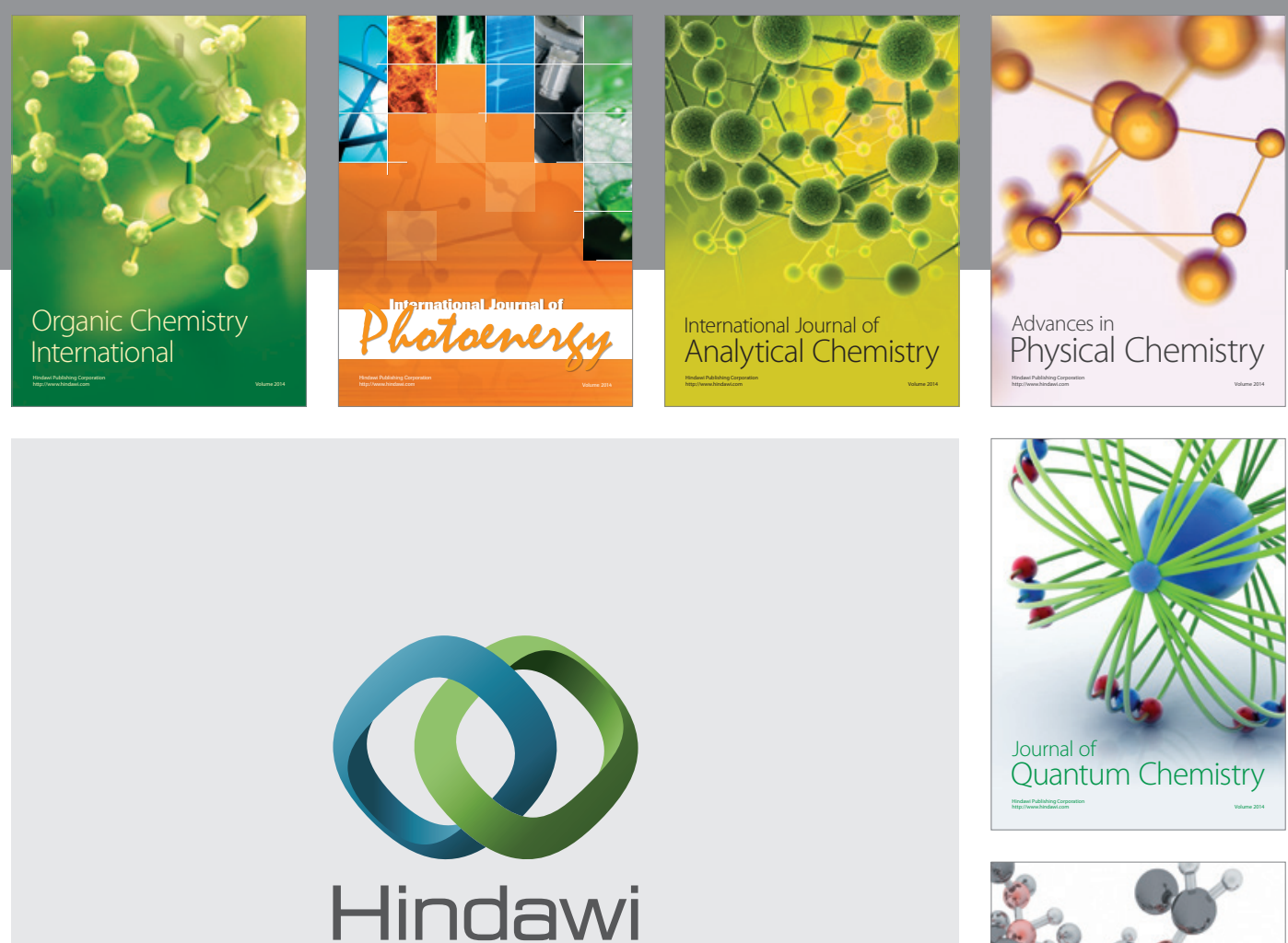

Submit your manuscripts at

http://www.hindawi.com

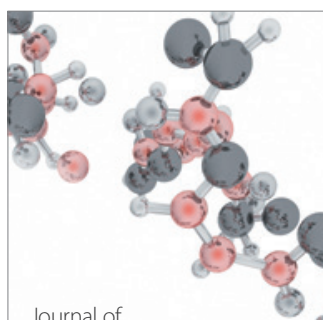

Analytical Methods

in Chemistry

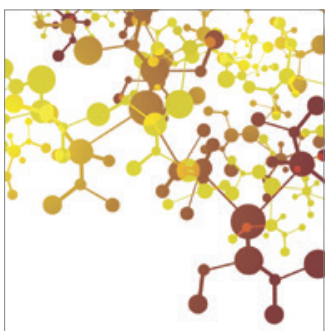

Journal of

Applied Chemistry

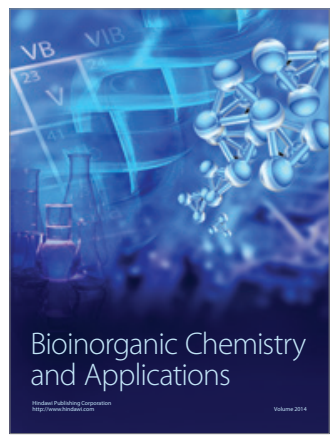

Inorganic Chemistry
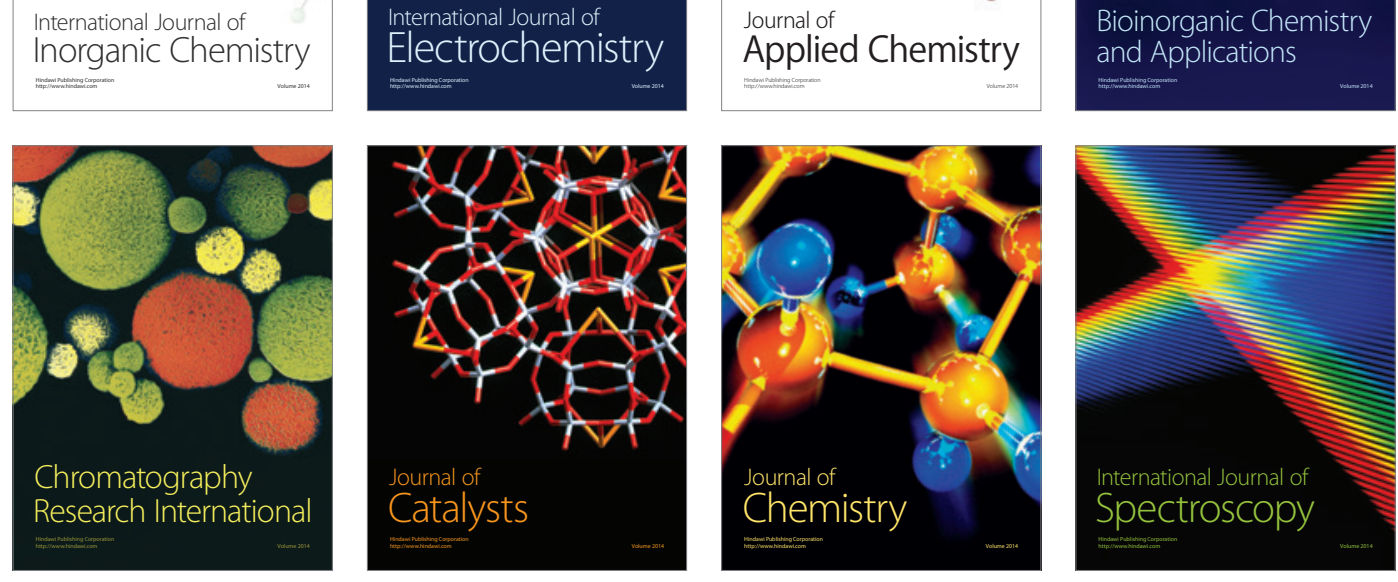